\title{
EVALUATION OF AXILLARY RESPONSE TO NEOADJUVANT CHEMOTHERAPY IN LUMINAL BREAST CARCINOMA TREATED IN CAISM- UNICAMP BETWEEN 2013 AND 2018
}

Pedro Lavigne de Castello Branco Moreira', César Cabello dos Santos¹, Renato Zocchio Torresan¹, Fabrício Palermo Brenelli', Susana Oliveira Botelho Ramalho'

${ }^{1}$ Universidade Estadual de Campinas - Campinas (SP), Brazil.

Introduction: Breast cancer is the most common malignant neoplasm affecting the female gender (besides non-melanoma skin cancer). It is a heterogenous disease with different phenotypic subtypes; the most common subtype is the luminal-like, which presents poor response to neoadjuvant chemotherapy. If patients with positive axilla at diagnosis are submitted to surgery, axillary dissection must be carried out, which is a surgery with major morbidities; however, if the patients are treated with neoadjuvant chemotherapy and develop negative axillary disease, they can avoid axillary dissection. Objective: to assess axillary response to neoadjuvant therapy in patients with cT1-3 cN1-2 luminal-like breast cancer. The secondary objectives were to assess the association between the axillary response to neoadjuvant chemotherapy according to: tumor replication marker (Ki67), estrogen and progesterone receptors (ER and PR), tumor histological grade, according to the Nottingham classification, tumor size (cT), level of axillary compromise (cN1, cN2 or cN3), chemotherapy scheme, luminal subtype and epidemiological variables (age, BMI, menopause status). Method: reconstituted cohort including female patients diagnosed with invasive breast cancer stage cT1-3 cN1-2 M0 at physical or ultrasound examination, who received neoadjuvant chemotherapy. Axillary compromise can be assumed. The patients were followed-up at the ambulatory of Clinical Oncology and Mastology at CAISM UNICAMP. A convenience sample was used.

Statistical analysis: Statistical analysis will be carried out using the Statistical Package for the Social Sciences, version 22.0 (SPSS). Correlations between categorical variables will be analyzed with the chi-square test. Differences between means will be verified using Student's t-test. Nonparametric tests will be used according to necessity. All tests will be bicaudal, with 5\% as the threshold of statistical significance. Results: One hundred and forty three cases were included, respecting the inclusion criteria. Of these, $2.8 \%$ evolved with pathological complete response per se (pCR); $5.6 \%$, with pCR in the breast; and $23.1 \%$, with axillary pCR. The lower the axillary compromise at diagnosis, the higher the frequency of axillary pCR (cN1 26.7\%, cN2-3 11.1\% - p=0.049). The smaller the residual lesion in the breast after chemotherapy (ycT), the higher the chances of axillary pCR (ycT0 28.8\%, ycT1 38.5\%, ycT2 9.7\%, ycT3-4 0 cases - p=0.042). The anthropometric, immunohistochemical and anatomopathological parameters did not present statistical relevance. Conclusion: Patients with luminal-like breast cancer and axillary compromise at diagnosis may benefit from avoiding dissection in about 20\% of the time if treated with neoadjuvant chemotherapy, so this therapeutic strategy should be considered in these cases. 